\title{
LA GOBERNANZA DE INTERNET COMO PLATAFORMA PARA IMPULSAR POLÍTICAS EN LA EDUCACIÓN CON TIC. El CASO de COLOMbia
}

\author{
Marcela Palacio Puerta* \\ Karen Isabel Cabrera Peña**
}

\section{Resumen}

Internet ha influido en todos los aspectos de la vida humana y la educación no es ajena a esta influencia. Sin embargo, cualquier aspecto relacionado con internet está en evolución. Este es el caso de la incorporación de tecnologías en la educación, cuyo desarrollo y medición están todavía en proceso de perfeccionamiento. La gobernanza de internet puede jugar un papel importante en dicho proceso ya que a través de ella se podrían implementar más y mejores políticas de educación con TIC, pues permite tener en cuenta todos los actores que se ven directa o indirectamente involucrados en ella.

Palabras clave: educación, tecnologías de la información y la comunicación, internet, gobernanza, políticas.

\section{INTERNET-LED GOVERNANCE: A TOOL TO PROMOTE ICT EDUCATION POLICY IN COLOMBIA}

\begin{abstract}
The Internet has influenced all aspects of human life, and education is no stranger to this influence. However, all aspects related to the Internet are still evolving. This is the case of the incorporation of technologies in education whose development and measurement are still in the process of improvement. Therefore, Internet governance can play an important role in this development because through it, there are more and better Information and Communication Technology (ICT) education
\end{abstract}

* Doctora en derecho, American University Washington College of Law. Docente investigadora, Universidad Sergio Arboleda, Bogotá (Colombia). [marcela.palacio@usa.edu.co].

** Doctora en Derecho, Universidad del Rosario. Docente investigadora, Universidad del Norte, Barranquilla (Colombia). [cabrerak@uninorte.edu.co].

Recibido: 7/03/2017/ Modificado: 15/05/2017/ Aceptado: 1/06/2017.

Para citar este artículo

Palacio Puerta, M. y Cabrera Peña, K. I. (2017). La gobernanza de internet como plataforma para impulsar políticas en la educación con Tic. El caso de Colombia. Opera, 21, pp. 5-23.

DOI: https://doi.org/10.18601/16578651.n21.02 
policies, taking into account all the direct or indirect key actors involved.

Key words: Education, information and communication technologies, internet, governance, policy.

\section{INTRODUCCIÓN}

Internet ha influenciado todos los aspectos de la vida humana y la educación no es ajena a esta influencia. No obstante, cualquier aspecto relacionado con la red se encuentra en proceso de evolución debido al sinnúmero de discusiones que existen a nivel global acerca de la regulación de contenidos digitales y de las tecnologías de la información y las comunicaciones (TIC) (Lucci, 2014).

La incorporación de las Tic en la educación, proceso que se ha venido desarrollando a nivel global promovido por organizaciones internacionales y gobiernos locales, todavía está en desarrollo. A pesar de lo anterior, existe consenso acerca de su importancia como herramienta para la promoción de la educación por diferentes razones que se estudiarán más adelante.

La gobernanza de internet, un fenómeno relativamente reciente, parte de la premisa de este -como herramienta global-no puede ser controlado o regulado por una sola entidad o gobierno. De esta forma, internet aparece como un espacio pluralista y democrático de discusión, donde múltiples agentes interesados, en los diferentes niveles -internacional, regional y nacional-, se reúnen en aras de promover políticas públicas o buenas prácticas que guíen su evolución.
Esta investigación sostiene que dichas características especiales de la gobernanza de internet brindan el escenario ideal para lograr que en países como Colombia se promuevan más y mejores políticas relacionadas con el uso de las Tic en la educación, ya que ofrecen el contexto para que de manera activa y permanente los actores interesados participen en su desarrollo, situación que no se presenta durante la práctica en la elaboración de políticas públicas, como se explicará en este artículo.

A pesar de los estudios existentes acerca de la importancia de las Tic en la educación, este artículo viene a contribuir al desarrollo sobre la materia al describir y explicar la relación y la importancia de la gobernanza de Internet para la incorporación o mejora de las políticas de uso de las Tic en la educación, particularmente en Colombia.

Este artículo, con el fin de presentar los resultados de esta investigación, se referirá, primero, al significado de incorporar las TIC en la educación y su importancia; segundo, a las políticas de las TIC en la educación en Colombia; tercero, analiza la gobernanza de Internet, y cuarto, los avances en el país sobre el tema. Posteriormente abordará cómo un papel más activo en el uso de la gobernanza de internet puede llegar a promover las políticas de la educación TIC en Colombia. Por último, se presentan las conclusiones de la investigación.

\section{METODOLOGÍA}

A fin de entender cómo ha influido la gobernanza en los últimos años en la orientación, calidad y eficacia de la educación a través de las TIC, esta investigación ha sido desarrollada 
desde el punto de vista analítico en el sentido de que a través de la descomposición en los elementos constitutivos (Lopera, Ramírez, Zuluaga, Ortiz, 2010, p. 17) de la gobernanza como fenómeno político, jurídico y social se pretende dilucidar la importancia de su intervención en la gestión del internet y los efectos en el campo de la educación.

Según el método analítico, la descomposición de los elementos constitutivos conlleva una serie de procesos para lograr la aproximación o solución de una problemática, que son: entender, criticar e incorporar (Caplow y Müller, 1946). En el caso de esta investigación se estudia, a través de estos procesos, y teniendo en cuenta datos históricos de su desarrollo, la manera como la gobernanza en Internet puede llegar a influir en la calidad y cobertura de la educación mediante TIC, particularmente en Colombia.

Aterrizando lo anterior, este documento primero recoge el análisis de cómo en Colombia se han venido incorporando en los últimos años las Tic en la educación y cuál ha sido el compromiso del país en el tema. Para este fin, se explica -a través de algunas políticas públicas que han sido implementadas a nivel nacional y municipal- el desarrollo y algunas problemáticas que aún faltan por abordarse.

Segundo, se analiza la gobernanza, a través de datos históricos, para interpretar cómo se ha desarrollado y cómo funciona en el uso y acceso de internet. Este aparte toma relevancia pues para criticar la participación de Colombia en la gobernanza de internet, que es el tercer segmento de este documento, es importante entender las nociones básicas de la figura de gobernanza y su desarrollo en la red.
Tercero, a partir del análisis de la incorporación de las TIC en educación y la participación de Colombia en la gobernanza de internet, por medio de la comparación, se presentan algunas apreciaciones sobre las incorporaciones que podría hacer Colombia en materia de gobernanza de internet para seguir desarrollando e incentivando las políticas de TIC en la educación.

Finalmente, como conclusiones, este aparte explica que en Colombia, a pesar de la claridad que existe sobre las ventajas de las TIC en la educación, las políticas implementadas sobre el tema podrían ser más y mejores si fueran parte del desarrollo de la gobernanza de Internet y si la tuvieran en cuenta como un medio y herramienta para promover y mejorar la educación.

\section{LAS TIC EN LA EDUCACIÓN}

Internet juega un papel importante en la educación, especialmente en las nuevas generaciones donde los estudiantes son nativos digitales, es decir, personas que no conocen un mundo sin PC, tabletas o internet (Kalaš et al., 2012). En este orden de ideas, la incorporación de internet en la educación no se limita a abrir las puertas a una amplia gama de materiales educativos, a reemplazar versiones impresas de texto por versiones digitales o a utilizar portátiles como herramientas de productividad (UNESCO, 2002b); la utilización del internet en la educación conlleva hacer parte integral del proceso educativo (UNESCO, 2002a), y, consecuentemente, la incursión en un nuevo modelo de aprendizaje, unas nuevas metodologías de 
la enseńanza y la utilización de nuevos contenidos (unesco, 2002a).

Dada esta transformación que propende el uso de las Tic en la educación, incluso, se ha empezado a utilizar el término de "Edupunk" (que significa hágalo usted mismo) para referirse a la enseñanza con internet (Fino, 2010), reflejando la esencia del nuevo modelo de enseńanza con Tic, donde los estudiantes asumen un rol activo de creadores de conocimiento (UNESCO, 2002a), y el docente de facilitador o coaprendiz (Anderson, 2010). Todo lo anterior dirigido a la creación de conocimiento de manera colaborativa.

La transformación del proceso educativo para lograr incorporar un modelo basado en la creación y el aprendizaje se da gracias a las nuevas herramientas Web 2.0 como wikis, blogs, plataformas para compartir videos como YouTube, entre otros, que permiten acceder, compartir y transformar conocimiento (Anderson, 2010). De igual forma, con estas herramientas se puede trabajar de manera conjunta con varios intervinientes, promoviendo así la colaboración.

El uso del internet en la educación y sus nuevas herramientas generan la creación de actividades y metodologías de aprendizaje para todos los niveles educativos y todas las áreas del saber. Por ejemplo, en las escuelas de Derecho de universidades como Harvard y Stanford se ofrece alojamiento de blogs a sus estudiantes, entendiendo el importante papel que pueden tener estas herramientas web 2.0 para la elaboración de la doctrina jurídica, lo que se denomina blaws ( blog + law) (Fino, 2010).

Por otro lado, en Nueva Zelanda, en las escuelas primarias, los profesores utilizan las facilidades de las páginas web para compartir con los padres de familia, niños y profesores de otras instituciones educativas los trabajos elaborados en clase (Kalaš et al., 2012). Igualmente, los profesores de escuelas americanas optan por utilizar videos de YouTube en clase y promover un aprendizaje más natural por parte de sus estudiantes (Light y Polin, 2010).

La incorporación de internet en la educación es una realidad y un propósito que se está promoviendo a nivel internacional, regional y nacional, estableciendo políticas que buscan la incorporación de la tecnología en la educación. Algunos ejemplos son el World Links for Development Programme (WorLD) del World Bank, una organización internacional que busca tomar provecho de las tecnologías para alfabetizar las poblaciones más vulnerables del mundo (World Bank, s.f); o la Agenda Digital para Latinoamérica y el Caribe, eLac2018, que es una agenda regional que promueve la utilización de las Tic como herramienta de inclusión social y, en concreto, pretende la incorporación de dichas tecnologías en la educación (CEPAL y Naciones Unidas, 2015). Finalmente, iniciativas locales como "Educando" en la República Dominicana (Educando, s.f) o "Escuelas del Futuro" en Guatemala (Ministerio de Educación, s.f.) que también buscan apoyarse en la tecnología para mejorar la calidad de sus sistemas educativos.

Existen diversas razones para incorporar las Tic en la educación, pero especialmente se pueden resaltar: 1) los nativos digitales, que son los estudiantes de hoy, requieren de un sistema educativo que refleje dicha realidad y brinde las habilidades requeridas (Kozma y Wagner, 2005); 2) la actual economía se basa 
en el conocimiento, y por tanto, los gobiernos ven la necesidad de educar ciudadanos que sean capaces de consumir y de producirlo y la utilización de las Tic juega un papel fundamental (UNesCo, 2005). Ahora, en los países en vía de desarrollo, estos justifican la búsqueda de la incorporación de las TIC en la educación en aras de solventar problemas tales como calidad y acceso (Rossini, 2010).

\section{POLÍTICAS DE TIC EN LA EDUCACIÓN EN COLOMBIA}

Colombia ha venido desarrollando las TIC en la educación con el propósito de transformar su sistema educativo, y dar solidez y apoyo a sus políticas de cobertura, calidad y eficiencia a nivel prescolar, básica, media y superior (Programa Nacional de Nuevas Tecnologías, s.f). En este sentido, el país ha participado y tomado lineamientos para desarrollar su política de Tic en la educación (Rendón, 2012) de las Cumbres Mundiales sobre la Sociedad de la Información celebradas en Ginebra en 2003 y en Túnez en 2005, donde se comenzó a plantear la importancia de las TIC para promover el desarrollo social y, entre otras cosas, para promover una educación de calidad y favorecer el proceso de aprendizaje y la alfabetización (CMSI, 2004).

De esta manera, Colombia traza el marco de la política nacional de TiC en la educación en diferentes planes tales como el Plan de Desarrollo 2010-2014, Plan Ministerio de las TIC, Plan Decenal de Educación 2006-2016 y el Plan Sectorial de la Educación 2010-2014 (Rendón, 2012). En este último se consolidó el Sistema Nacional de Innovación Educativa que estableció como objetivo, entre otros, promover el uso y la apropiación de las tecnologías de la información y la comunicación (TIC), y hacer de estas su principal soporte. Asimismo, se establecieron los objetivos a nivel regional sobre la utilización de Tic en la educación (Ministerio de Educación Nacional, s.f).

El Sistema Nacional de Innovación planteó estrategias en diferentes campos de acción, y se ha dirigido hacia la total incorporación de las TIC en la educación, yendo más allá de simplemente entregar computadores a los colegios sin tomar las medidas necesarias para lograr un uso efectivo de estas tecnologías como sucede en otros países (UNEsco, 2014). En este sentido, dicho sistema trazó metas en aspectos como acceso a la tecnología, gestión de contenidos digitales, formación de docentes en uso de las TIC y apropiación pedagógica de estas y promoción en la investigación y uso de contenidos digitales (Ministerio de Educación Nacional, s.f).

Los planes de incorporación de TIC en la educación se han ido desarrollando a través de diferentes programas. Por ejemplo, el aumento de acceso a las TIC se ha desarrollado por medio de programas como Computadores para Educar, Compartel y Kioskos vive digital, que buscan proveer con hardware y conectividad a las escuelas y a la población (Programa Nacional de Nuevas Tecnologías, s.f; MinTic, 2014). El trabajo del Gobierno colombiano en el desarrollo de estos objetivos lo llevó a que Computadores para Educar ganara, en el 2012, en la Cumbre Mundial sobre la Sociedad de Información, el premio a mejor programa mundial en la categoría de acceso a la información y al conocimiento (MinTic, 2014). 
Por otro lado, la creación de contenidos y la promoción de metodologías colaborativas se ha llevado a través del portal Colombia Aprende (Programa Nacional de Nuevas Tecnologías, s.f), el cual tiene un doble propósito, promover la generación de contenidos y servir como la primera comunidad de aprendizaje colaborativo en Colombia. Finalmente, la acción de formación docente y desarrollos pedagógicos con Tic ha avanzado a través de iniciativas como la campaña ¡A qué te cojo ratón! y Computadores para Educar (Ministerio de las Comunicaciones, 2008).

Regionalmente, también se han desarrollado políticas con miras a la incorporación de las TIC en la educación. Diferentes planes de desarrollo actuales lo incluyen como un componente con miras al mejoramiento en el acceso y la calidad de la educación, no obstante, la proyección del uso de las TIC en la educación varía según el nivel de acceso a las tecnologías de las regiones. Por ejemplo, Antioquia, en su Plan de Desarrollo 2016-2019 "Antioquia piensa en grande" establece, en su línea estratégica de Equidad y Movilidad Social, como prioridad incorporar las TIC en procesos educativos, cambiando la forma de pensar y aprender, es decir aspirando a una transformación del sistema educativo a través del uso de la tecnología (Gobernación de Antioquia, s.f).

Por otro lado Chocó, uno de los departamentos con mayor brecha digital, estableció en su Plan de Desarrollo 2016-2019 "Oportunidades para todas la subregiones”, en su Estrategia para el Desarrollo Institucional Propio, el programa Tic, en el cual se establecen como proyectos ampliar el uso de la infraestructura TIC en las instituciones públicas educativas a través del programa Computadores para Educar, la promoción de contenidos innovadores con el uso de las Tic en la educación y la formación de docentes en el uso de estas herramientas (Gobernación del Chocó, s.f.).

A pesar de los grandes esfuerzos del Gobierno nacional y territorial en la formulación e implementación de la política de TIC en la educación, y en algunos casos, de un pequeño segmento del sector privado y la sociedad civil, como en el caso de "Medellín Digital", promovida por la Alcaldía de Medellín y sus secretarías, la Fundación Empresas Públicas de Medellín, el Centro de Ciencia y Tecnología de Antioquia (sector privado) y la Universidad EAFIT por el sector educativo (Polanco, 2011), todavía falta camino para lograr esta incorporación de las TIC.

Dadas las características de una política pública con el objeto de incorporar las TIC en la educación, una participación activa de todos los involucrados en su formulación e implementación sería una gran herramienta de apoyo. Lo anterior se debe a que no solo se trata de la promoción de un derecho humano que requiere de una participación democrática activa (Crosso, 2013), sino que la incorporación de las Tic en la educación -por su naturaleza- requiere de una participación activa de todos los involucrados -estudiantes, docentes, instituciones educativas, padres de familia, Gobierno, servicios del sector privado, entre otros-, y por tanto, estos deben ocupar un papel permanente en dicha formulación e implementación.

En este orden de ideas, la legislación colombiana ha intentado promover un ambiente participativo para la gestión pública. Por ejemplo, el preámbulo de la Constitución 
Política de 1991 establece que en Colombia se respetarán los derechos "dentro de un marco jurídico, democrático y participativo", lo que demuestra el papel activo que se busca otorgar a los ciudadanos en el contexto colombiano. De igual manera, y desde la educación, la Ley 115 de 1994 regula la participación ciudadana en los procesos de formación de políticas públicas en el tema, otorgándoles voz a los interesados. Desde el punto de vista institucional, el Ministerio de Educación ha puesto a disposición algunos espacios para la participación ciudadana como los foros educativos municipales (Ministerio de Educación, s.f.b).

No obstante, a pesar de dicha estructura normativa, lo cierto es que la participación ciudadana pocas veces se lleva a cabo o tiene efectos en las políticas públicas de educación ya sea por injerencia de actores políticos y sus intereses particulares (Jiménez, 2012), por tratarse de un sistema de participación de creación estatal sin concertación de la ciudadanía (Velásquez y González, 2003), o por falta de generalización de las iniciativas participativas por ausencia de modificación de procesos administrativos (Arias et al., 2015), entre otras. Teniendo en cuenta este panorama, no es ilógico afirmar que la formulación e implementación de las políticas de TiC en la educación no han contado con una activa participación de la sociedad civil, afirmación sustentada además por la falta de documentación sobre su rol en el proceso.

\section{LA GOBERNANZA DE INTERNET}

Antes de los años ochenta, los Estados eran los únicos encargados del desarrollo social y económico de sus ciudadanos. Con la entrada de la tecnología y los procesos de globalización, estos últimos se han visto incentivados a intervenir de manera más directa en los asuntos atinentes a la libertad y la reivindicación de sus derechos (Mayntz, 2001). Estos procesos, donde la sociedad civil asume un rol más determinante en las decisiones del Estado, se conoce como gobernanza (Instituto de investigación y debate sobre gobernanza, 2016).

Considerando lo anterior, la gobernanza propende por un equilibrio entre el Estado y la sociedad civil en su interacción en la economía y la política, y en aspectos sociales como los derechos humanos asociados a asuntos ambientales y tecnológicos. La idea principal es, ante las falencias de la actividad gubernativa, ampliar la capacidad del Estado con enfoques que vengan de la ciudadanía, y particularmente de actores sociales que son quienes viven y conocen las problemáticas sociales (Mayntz, 2001), para que este llegue a ser legítimo, competente y eficaz.

Según el Programa de las Naciones Unidas para el Desarrollo (PNUD), que busca erradicar la pobreza, la exclusión y la desigualdad en el mundo, la gobernanza es un proceso de gobierno que activa el poder público, los mercados y las redes sociales en un sistema de coordinación mediante iniciativas de diálogo, discusión, entendimientos, negociaciones, acuerdos y compromisos con organizaciones privadas y sociales, para la implementación de políticas y programas sobre derechos humanos, proyectos de inversión y prestación de servicios (Aguilar, 2010).

Teniendo en cuenta la definición del PNUD, la comunidad de las Naciones Unidas 
considera que una gobernanza logra sus fines cuando las instituciones y los procesos dentro de los Estados son transparentes y promueven la participación, el pluralismo y la equidad (Naciones Unidas, 2016).

En el caso de internet, que es una red que genera gran intercambio de información entre las personas, por ser un medio rápido y poco costoso (Garrote, 2003), es necesario que se administre y proteja. Debido a la pluralidad de agentes que intervienen en la red, la gobernanza ha sido el canal para procurar el desarrollo de reglas y procesos entre los gobiernos, el sector privado y la sociedad para regular su uso y evolución (Kurbalija y Gelbstein, 2005).

En este sentido, la gobernanza en internet busca fomentar la participación colectiva de las personas en los procesos de comunicación y comercialización que pueden llegar a fomentar la innovación, el acceso a la información y a la educación (Schmitz, 2005), pues su carácter descentralizado hace que los contenidos y la información que se transmite pueda ser recibida y enviada -de manera interactivapor cualquier persona en cualquier lugar del mundo (Esteve, 2006).

Ahora bien, aunque las TIC han posicionado el conocimiento y la cultura como un recurso esencial que va por encima de los factores de producción y que pueden ser comercializados, e internet permite inmediatez y disponibilidad de espacio para que salga a la luz la información y se hagan cualquier tipo de transacciones e intercambios (Rengifo, 2008), aún no hay una cobertura total y completa de acceso a la red en el mundo.

La falta de conexión de las personas a internet puede disminuir su desarrollo social y económico, además minimiza los recursos y las oportunidades de las que sí gozan las personas que tienen acceso a esta red, afectando, en gran o poca medida, sus derechos fundamentales. Por ejemplo, sin internet no se conoce, de manera tan expedita, información y sucesos políticos, económicos y sociales que ocurren alrededor del mundo, es decir, el derecho a la información se ve truncado; y las expresiones creativas o culturales de quienes no acceden a la red no podrán llegar a tantas personas como los que tienen acceso, afectándose el derecho a la cultura y la libre expresión (Kurbalija y Gelbstein, 2005), por lo que contar con este recurso se convierte en una oportunidad para avanzar socialmente.

En algunos lugares, las poblaciones no pueden hacer uso de las ventajas de internet y las TIC debido a que no cuentan con los recursos económicos para pagar el servicio, se encuentran alejadas de las urbes, en lugares donde hay cobertura, no existe la infraestructura de telecomunicaciones o no tienen la educación para entender cómo funciona la red (Padilla, 2012). En este sentido, la gobernanza se hace necesaria para permitir la conectividad, minimizar la fragmentación de internet, salvaguardar y proteger los derechos de los usuarios, definir las responsabilidades de las diferentes partes que interactúan en la red y evitar la mala utilización o abuso de la información en ella contenida (Kurbalija y Gelbstein, 2005).

En otras palabras, las cuestiones antes señaladas podrían solucionarse con políticas efectivas que aborden las problemáticas que no permiten que todas las personas estén conectadas o que lo hagan con una calidad muy baja de internet; de esta manera, ante la falta 
de medidas efectivas y eficaces que tengan en cuenta los contextos políticos, sociales y económicos, las entidades privadas y públicas, y sobre todo la sociedad civil, han tenido que intervenir por medio de la gobernanza.

Siguiendo con el carácter descentralizado de internet, es relevante acotar que no es posible su completo control por parte de una institución, lo que implica que hablar de su gobernanza se refiere a todas las decisiones que se pueden llevar a cabo y que inciden en él (Galindo, 2012). Estas decisiones están en constante evolución debido a que las TIC también avanzan a pasos agigantados y, adicionalmente, provienen de sectores tan diversos como el académico, la comunidad técnica, autoridades regulatorias de comunicación, fundaciones prolibertad de expresión y muchos más.

Aunque, como se estudió en las líneas anteriores, la gobernanza en internet aborda varias problemáticas, han sido la neutralidad y el acceso, quizás, los tópicos que más se han desarrollado y más retos implica. La neutralidad se refiere a la garantía que debe tener cualquier persona de poder consultar los contenidos que desee sin discriminación, sin cambios en la calidad o intromisión en el tipo de contenido o servicio con el que cuenta (Pérez y Olmos, 2009), mientras que el acceso tiene relación con la asequibilidad, disponibilidad y calidad de internet para que los ciudadanos puedan usar y participar en este (Galindo, 2012).

Sin neutralidad en la red, quienes prestan el servicio de acceso, conocidos como proveedores de servicios de internet (PSI), pueden impedir que los usuarios visiten ciertos sitios web o reducir la velocidad para incrementar el tráfico hacia otros sitios (Fernández, 2001).
En consecuencia, la gobernanza promueve políticas públicas y leyes que posibiliten a las poblaciones expresarse libremente en línea y acceder a cualquier tipo de información sin condicionamientos sociales o políticos (Marsden, 2012). Con medidas idóneas sobre neutralidad en la red ningún proveedor podrá bloquear o afectar el acceso a sitios web, noticias o foros.

El acceso a internet es una de las áreas más abordadas por la gobernanza debido a que ampliar la posibilidad de usar la red, mejorar la conectividad y en fin, reducir la brecha digital, es necesario para el desarrollo de la sociedad de la información si se tiene en cuenta que las interacciones sociales, económicas y políticas se llevan a cabo por este medio (Kurbalija y Gelbstein, 2005). La idea con la gobernanza sobre acceso es asegurar que la red sea asequible, garantizar conexión en ciudades, sean grandes o pequeńas; desarrollar redes troncales e infraestructura para que internet llegue a zonas rurales, pues el intercambio de ideas y la difusión del conocimiento por este medio permiten la conservación de los derechos a la cultura y a la educación, y el progreso, en general, de la ciencia (Padilla, 2012).

Como se observa a lo largo de este apartado, la gobernanza puede llegar a ser un medio para que las partes interesadas participen activa y directamente en los debates que se susciten respecto a este tema. En el caso de internet, el solo acceso a este, o la implementación de Tic, sin tener en cuenta contextos sociales, políticos y económicos, no permite la cobertura total y completa a esta red, y es por esta razón que las discusiones que se han desarrollado acerca de la gobernanza en internet toman gran relevancia 
en las políticas que adoptan los Estados sobre el tema.

\section{Algunas apreciaciones sobre su evolución en el ámbito internacional}

Para la época de los años ochenta, la mayoría de la comunidad mundial ya utilizaba Internet, por lo cual, al igual que este se iba desarrollando, se iba evidenciando la necesidad de una regulación que delimitara los estándares de su uso y acceso (Kurbalija y Gelbstein, 2005). Es así como nace en 1986 una comunidad internacional, sin intervención de los gobiernos, denominada Fuerza de Tareas de Ingeniería para Internet (IETF por sus siglas en inglés) que procura regular las propuestas y los estándares de internet (Internet Society, 2017).

En el año 1994, la Fundación de las Ciencias de Estados Unidos involucró en la IETF a la empresa Network Solutions que administraba el Sistema de Nombres de Dominio (DNS, por sus siglas en inglés) y representaba al sector privado. Las demás integrantes de la comunidad no vieron con buenos ojos la llegada de Network Solutions por lo que, después de desacuerdos y discusiones sobre las partes que deberían ser integrantes de la IETF, en el año 1998 se estableció la Ministerial Meeting on Digital Economy, Internet Corporation for Assigned Names and Numbers (ICANN), en donde el debate sobre gobernanza de Internet ha tomado mayor relevancia y tenido mayor participación de los gobiernos a través de las Naciones Unidas (ICANN, s.f).

Finalmente, de los esfuerzos de la ICANN, la Unión Internacional de Telecomunicaciones y las Naciones Unidas, en el año 2003, se realizó la Cumbre Mundial de la Sociedad de la Información (CMSI) en donde se insta a los países a incorporar dentro de sus agendas diplomáticas la gobernanza en internet (Organismo especializado de las Naciones Unidas para las Tic, 2006). Entre muchos de los temas desarrollados, se propone el establecimiento de un grupo de trabajo sobre gobernanza de internet (GTGI).

La idea de constituir dicho grupo se basa en que para coordinar la gobernanza en internet, por medio de un proceso abierto e inclusivo, es necesario un escenario que permita total y activa participación de los gobiernos, el sector privado y la sociedad civil de todos los países del mundo sin importar su desarrollo (Organismo especializado de las Naciones Unidas para las TIC, 2006). Por medio del GTGI, entonces, se crea el espacio para dialogar y debatir las propuestas sobre gobernanza de internet.

Las reuniones del GTGI han dado lugar a varios avances sobre el tema. Por ejemplo, se ha desarrollado una definición sobre gobernanza en internet que hace énfasis en la necesidad de que las reglas y los procedimientos del uso de internet sean resultado de las decisiones comunes entre gobiernos, sector privado y sociedad (Kurbalija y Gelbstein, 2005). También ha colaborado a que se desarrolle un entendimiento común sobre cómo la gobernanza en internet propende por la protección de derechos humanos como la educación, el derecho a la información, el derecho a la privacidad y la libertad de expresión.

Sobre este último punto, el GTGI ha concluido que los múltiples usos y recursos de las TIC e internet pueden, por un lado, promover la salvaguarda y protección de los derechos 
humanos consignados y, por el otro, pueden llegar a ser un impedimento para el ejercicio de los mismos, y es por esta razón que la gobernanza cobra relevancia (Kurbalija y Gelbstein, 2005). Al respecto, en el año 2012, el Consejo de Derechos Humanos de la onu, en el $20^{\circ}$ periodo de sesiones sobre la promoción y protección de todos los derechos humanos, civiles, políticos, económicos, sociales y culturales, incluido el derecho al desarrollo (Naciones Unidas, 2012), menciona que la protección de los derechos humanos debe ser, en cualquier medio, lo que incluye las plataformas tecnológicas como internet.

Una muestra sobre cómo el GTGI ha influenciado en la gobernanza de internet en algunos países es, por ejemplo, en Estados Unidos, la Comisión Federal de Comunicaciones (FCC) que adoptó tres principios para preservar el internet libre y abierto: primero, el derecho de los usuarios a acceder a contenidos legales en la red; segundo, el derecho a utilizar aplicaciones y servicios según sus necesidades $o$ gustos y, por último, otorgar la posibilidad de conectarse a Internet según el dispositivo y proveedor que desee. Debido a que esta comisión es una agencia independiente del Gobierno de Estados Unidos y bajo responsabilidad directa ante el Congreso, aún no hay consenso general sobre si estos principios son suficientes para la protección de internet (Federal Communications Commission, 2016).

Otros instrumentos que vale la pena recalcar son los principios para la Gobernanza y el uso de internet del Comité Directivo de Internet de Brasil (CGr.br) y la Declaración del Comité de Ministros sobre los principios para la gobernanza de internet del Consejo de
Europa. En el primer caso, este comité brasileño, que cuenta con representación del Gobierno, el sector corporativo y la academia, por medio de estos principios procura promover la calidad técnica, la innovación y la difusión de servicios disponibles en la red (Comité Gestor da Internet no Brasil, 2016). Por su parte, la Declaración del Consejo de Europa de 2011 destaca 10 principios entre los que se encuentran la universalidad de internet, la protección de las partes interesadas en las actividades que se realizan en la red y la gestión descentralizada del mismo (Council of Europe, 2011).

\section{COLOMBIA Y LA GOBERNANZA DE INTERNET}

Colombia no ha sido extraña a la tendencia internacional y regional de buscar espacios de discusión y encuentro entre la sociedad civil, el Estado, los empresarios y la academia para el debate sobre la masificación del uso del internet y de los asuntos de importancia relacionados con este, como los aspectos técnicos y de infraestructura; el papel y las responsabilidades de los diferentes actores; la economía de la conectividad; los derechos humanos; y propiedad intelectual (Comisión de Regulación de Comunicaciones, 2015). Es decir, Colombia es un país activo en el uso e implementación de la gobernanza de internet y es, junto a México y Brasil, uno de los líderes en la región en los debates sobre el tema (Ministerio de las Tecnologías de la Información y las Comunicaciones, 2015).

Los avances en las discusiones sobre gobernanza en internet, que habían comenzado 
a nivel internacional a partir del 2003 impulsados por las Naciones Unidas, promovieron que dicho escenario de discusión se abriera en Colombia. En el 2013 se creó "Fgicolombia" grupo de trabajo en torno a la gobernanza de internet en Colombia que buscaba abrir espacios para que la comunidad de la red se pudiera incorporar a las discusiones sobre el tema.

Este grupo se conformaba por actores de diferentes medios, lo que se denomina en inglés un grupo multi-stakeholder, que posteriormente conformó la Mesa Colombiana de Gobernanza de Internet y ha participado activamente en diferentes reuniones globales y regionales como el Latin American and Caribbean Internet Governance Forum (LaCIGF5), Internet Governance Forum (IGF) Bakú, Lacigf6, Córdoba Argentina, Igf Bali, Lacigf7 San Salvador y el Igf8 Estambul (Meneses, s.f.).

De esta forma, en Colombia la característica de ambiente de discusión pluralista de la gobernanza de Internet se ha implementado gracias a la participación de diversos actores de varios sectores de la sociedad que confluyen en la Mesa de Gobernanza de Internet con diferentes intereses. Por ejemplo, los miembros de la sociedad civil, como el observatorio de la Juventud, Mozilla Colombia, AGEIDA DENSI Colombia, Colnodo, Fundación para la Libertad de Prensa (FLIP), Fundación Karisma, la Asociación Colombiana de Usuarios de Internet (ACUI), han buscado un internet con enfoque social.

Por su lado, los miembros de sector privado como Google Colombia, Intel Colombia, Twitter, Telefónica Colombia, co Internet SAS asisten a la gobernanza de internet con el obje- tivo de extender los servicios y el mantenimiento de la infraestructura de este. El sector de la academia, representado por la Universidad del Rosario, tiene el interés de investigar sobre los impactos sociales y culturales de las TIC; $y$ por último, el sector del Gobierno busca identificar los actores y las temáticas nacionales a través de la Comisión de Regulación de Comunicaciones (CRC) y el Ministerio de Tecnologías de la Información y las Comunicaciones (Mesa de Gobernanza de Internet, s.f).

La participación de estos actores en la mesa de gobernanza de internet ha sido activa tanto a nivel nacional como internacional. Por ejemplo, durante el 2016, el Gobierno colombiano participó en la OCDE, en el ICANN 56, y en el IGF en Guadalajara México (CRC, 2016). De igual manera, los insumos provenientes de los diferentes foros de internet son utilizados para la formulación de políticas públicas (CRC, 2016).

La reunión de actores tan diversos permite que se aborden temas de importancia para todos los sectores de la sociedad y desde distintos puntos de interés en búsqueda de la formulación de políticas públicas que beneficien a la sociedad y la apropiación de las TIC. Es así como en la Mesa de Gobernanza de Internet se discute sobre la libertad de expresión y privacidad, honra, violencia contra las mujeres en espacios digitales, ciberseguridad, acceso a la información, neutralidad en internet, problemas de una visión maximalista del derecho de autor (Gobernanza de Internet en Colombia, 2014), acceso para la inclusión, y desarrollo y equidad de género (Mesa Colombiana de Internet, 2016). 
Como se puede observar, actualmente este escenario diverso no ha centrado sus temas de discusión a la incorporación o uso de las TiC en la educación, aunque algunos temas puedan llevar a la formulación e implementación de políticas que favorezcan este proceso como el acceso a la información y la inclusión.

\section{UN PAPEL MÁS ACTIVO EN EL USO DE LA GOBERNANZA DE INTERNET PARA PROMOVER LA EDUCACIÓN CON TIC}

Actualmente, sobre las políticas públicas relacionadas con el uso de las TIC en la educación se encuentra que, por un lado, el Gobierno nacional ha mostrado un fuerte compromiso en su formulación e implementación, no obstante, dicha política todavía se encuentra en proceso de evolución y, a pesar que podría beneficiarse de la participación activa de la ciudadanía, poca ha sido su intervención por la misma naturaleza del sistema de participación ciudadana en políticas públicas.

Por otro lado, tanto el Gobierno como la sociedad civil y el sector privado han utilizado los foros y principios de gobernanza de internet para formular políticas en temas relacionados con el uso de las Tic de manera mancomunada y, además, para aprender y enriquecerse de la participación extranjera. Sin embargo, el tema de uso de las Tic en la educación ha sido inexplorado aunque podría ser el espacio que permita impulsar la política de TIC en la educación.

Las características que brinda la gobernanza de Internet podrían mejorar la formulación e implementación de políticas de Tic en la educación y, a su vez, lograr que la formulación sea más rápida. Por ejemplo, si se establece el uso de las Tic en la educación como un tema de trabajo en gobernanza de internet, con la participación activa y permanente de todos los interesados, se podrían identificar cuáles son los aspectos en los que todavía existen falencias y enfocar el desarrollo de las políticas públicas hacia la superación de dichas barreras.

En concordancia con lo anterior, este escenario es pertinente pues brinda un espacio abierto y permanente a actores como la academia y organizaciones sin ánimo de lucro, que anteriormente no tenían un papel favorecido en la realización de políticas públicas relacionadas con las nuevas tecnologías o la educación, como se mencionó, y quienes tienen un conocimiento y conexión directa con el escenario educativo y sus necesidades.

De igual manera, lo anterior favorece la formulación de políticas sobre el uso de las TIC en la educación ya que su incorporación requiere que todos los sectores involucrados actúen de manera conjunta para lograr transformar el sistema de aprendizaje, y, por tanto, su participación activa en la formulación de la política permitiría que se desarrollen teniendo en cuenta el rol específico de cada uno de los participantes del proceso educativo.

En este orden de ideas, la participación de actores locales en foros regionales e internacionales de gobernanza permite, primero, enriquecer las discusiones nacionales sobre las políticas necesarias para continuar promoviendo el uso de las Tic en la educación, y segundo, aprender de experiencias de otros países que están en un nivel diferente de incorporación de estas y beneficiarse de la realización de guías y buenas prácticas que desarrollan diversas organizacio- 
nes internacionales (Hassan, 2005), Es decir, la gobernanza de internet podría utilizarse como plataforma de impulso para el desarrollo y la mejora de las políticas de Tic en educación.

La propuesta de darle un papel principal a la gobernanza de Internet para promover la formulación o implementación de políticas públicas a fin de incorporar las Tic en la educación ya ha sido puesta en práctica por otros países, obteniendo resultados identificables. Por ejemplo, en iniciativas sobre educación transnacional y métodos de aprendizaje en línea, uno de los primeros intentos integrales de carácter internacional para asegurar la calidad de los programas educativos transnacionales es el "Código de buenas prácticas en la provisión de educación transnacional" de la unEsCo (Kurbalija y Gelbstein, 2005).

Este Código, que ha sido implementado por el Consejo Europeo en la Unión Europea y utilizado en otros países como Australia y Reino Unido, es el producto de diversas conversaciones entre los diferentes actores involucrados en la educación fronteriza y tiene como finalidad garantizar la calidad, independientemente del modelo de enseñanza, a través de recursos educativos en línea y abiertos, para preservar la integridad de la titulación académica (Knight, 2007).

En el caso del reconocimiento de los grados o títulos en procesos de aprendizaje en internet, también la gobernanza ha influenciado para que los reconocimientos sean válidos a nivel regional y mundial. Por ejemplo, el programa sócrates de la Unión Europea propugna por la cooperación de los integrantes en todos los ámbitos de la educación, y para lograr el reconocimiento de los cursos y progra- mas cursados en línea realiza periódicamente reuniones en donde se aprueban proyectos comunes de educación, se crean redes europeas para la difusión de ideas y prácticas correctas en aprendizaje, y se hacen estudios y análisis comparativos sobre los estándares educativos de los miembros (Eur-Lex, 2007).

En el ámbito local se pueden resaltar los materiales educativos para internet del Ministerio de Educación, Cultura y Deporte en España. Estos materiales, que han sido el resultado de las políticas implementadas por el Instituto Nacional de Tecnologías Educativas y de Formación del Profesorado, busca que los materiales educativos elaborados para la creación de recursos multimedia por medio de TIC, además de tener un carácter didáctico, sean accesibles y ajustados a los contenidos curriculares vigentes (Cabrera, 2014). Para este fin, es la misma comunidad, profesorado y estudiantes los que proponen los materiales y verifican la viabilidad de ser usados en el aula o en el hogar.

Como se observa, la gobernanza de internet se ha utilizado en beneficio de la educación con TIC. Teniendo en cuenta el fuerte rol que están jugando el Gobierno colombiano, la sociedad civil y el sector privado, al igual que la importancia de las políticas de las Tic en la educación para Colombia, sería beneficioso hacer uso de dicho escenario para promover, impulsar y mejorar las mencionadas políticas.

\section{CONCLUSIONES}

En los últimos años, con el auge de las nuevas tecnologías, internet ha influenciado 
todos los aspectos económicos, políticos y sociales de la vida humana. Debido al rápido y continuo avance de las formas de comunicación e interacción en la red, cualquier aspecto relacionado con este se encuentra en evolución; es así como los Estados están continuamente estudiando de qué manera estas nuevas formas de interacción pueden propender a la protección de los derechos humanos tales como la libertad de expresión y la educación.

La gobernanza, un fenómeno relativamente reciente, procura una forma más amplia de relación entre gobernantes y gobernados para solucionar y combatir problemas de toda índole en la sociedad, en el caso de internet lleva consigo el entendimiento de que, como herramienta global, esta no puede ser controlada o regulada por una sola entidad o Gobierno. De esta forma, la gobernanza se consolida como un espacio pluralista y democrático de discusión donde múltiples agentes interesados, en los diferentes niveles -internacional, regional y nacional-, se reúnen con el fin de promover políticas públicas o buenas prácticas que -en este caso-guíen la evolución de internet.

En consecuencia, lo gobernanza de internet favorece la promoción de derechos humanos como el de la educación al facilitar un ambiente democrático y pluralista de discusión que permite la participación de diversos actores interesados. Particularmente, en la educación ha logrado que sectores, entre ellos la sociedad civil y la academia -que anteriormente no jugaban un papel central- logren intervenir y aportar en la materialización de la educación como objetivo público a través de políticas públicas de acceso a la red, o acciones que permitan la utilización de esta para reducir la pobreza, entre otros.

Como lo demuestra esta investigación, cuando las discusiones de la gobernanza de internet se han centrado en torno a los derechos humanos como la educación y han tomado en cuenta las distintas opiniones de los sectores interesados en el asunto, ha sido posible hallar falencias en pro de mejorar las políticas existentes, así como también, al mismo tiempo, aprender de otras experiencias y buenas prácticas en las reuniones regionales e internacionales que se han llevado a cabo.

Teniendo en cuenta lo dicho, la gobernanza de internet se ha enfocado no solo en la promoción de educación a través del empoderamiento por las TIC por medio del impulso de políticas públicas dirigidas a este objetivo, sino que se ha consolidado como una herramienta que protege a los ciudadanos para que ninguna regulación o política que gire en torno a las tecnologías de la información y de la comunicación se convierta en una barrera para la educación.

En el caso de estudio de Colombia, donde el fenómeno de gobernanza de Internet es relativamente joven pero ha tenido mucho auge, se concluye que, a pesar de que el foro de gobernanza de internet nacional no ha abordado directamente el tema de incorporación de las Tic en la educación, las diversas actividades locales, regionales e internacionales que ha llevado a cabo y en donde ha participado han empezado a influenciar las políticas públicas del Estado colombiano hacia un camino que permite mejorar la utilización de las Tic en la educación. 
Por último, pero no menos importante, se concluye que, debido a la dinamicidad de internet y la tecnología, aún las políticas públicas que hay sobre la red, que procuran la cobertura mundial y la calidad real en la educación, se han quedado cortas para lograr el cometido, por lo que se necesita que la gobernanza en internet siga abriendo espacios de mayor debate e interacción entre los sectores interesados para que los avances sean acordes a las necesidades actuales.

\section{REFERENCIAS}

Aguilar, L. (2010). Gobernanza: los nuevos procesos de gobernar. México: Editorial Fundación Friedrich Naumann para la Libertad.

Anderson, J. (2010). ICT Transforming Education: a Regional Guide. Bangkok: UNESCO. Recuperado de http://unesdoc.unesco.org/ images/0018/001892/189216e.pdf

Arias, J. et al. (2015). La participación ciudadana en la implementación de las políticas educativas en Colombia, Cuadernos de Administración, 31 (53), 131-138. Recuperado de http://www.scielo.org. co/pdf/cuadm/v31n53/v31n53a12.pdf

Cabrera K. I. (2014). Modelos de acceso abierto en educación y ciencia. Revista Educación y educadores, 17 (2), 321-338.

Caplow T. y Müller A. (1946) Hacia una definición analítica de la sociología. Revista Mexicana de Sociología, 8 (3), 421-426.

Cepal y Naciones Unidas (2015). Agenda Digital para América Latina y el Caribe (eLAC2018). Recuperado de http://repositorio.cepal.org/ bitstream/handle/11362/38886/S1500758_ es.pdf?sequence $=1$ \&isAllowed $=y$
CMSI (2004). Declaración de Principios. Construir una sociedad de la información: un desafio global para el nuevo milenio (WSIS-03/GENEVA/4-S). Ginebra. Recuperado de http://www.itu.int/net/ wsis/docs/geneva/official/dop-es.html

Comisión de Regulación de Comunicaciones (20165). Gobernanza de Internet en Colombia (Informe No. 2). Recuperado de https://www.crcom.gov. co/recursos_user/2016/Informes/Informe_2_ GI_07_04_16.pdf

Council of Europe (2011). Declaration by the Committee of Ministers on Internet governance principles. Recuperado de https://search.coe.int/cm/Pages/result_details.aspx?ObjectID $=09000016805$ cc2f6

Crosso, C. (2013). La participación ciudadana en el debate y definición de la política pública. En Luna, M. (ed). Participación ciudadana, politicas públicas y educación (pp. 53-64). Quito: Contrato Social por la Educación.

Educando (s.f). Educando el Portal de la Educación Dominicana. Recuperado de http://www.educando.edu.do/

Eur-Lex (2007). Programa Sócrates. Recuperado de http://eur-lex.europa.eu/legal-content/ES/ TXT/?uri=URISERV\%3Ac11043

Esteve, L. (2006). Aspectos internacionales de las infracciones de derechos de autor en Internet. Granada: Comares.

Federal Communications Commission (2016). About FCC. Recuperado de https://www.fcc.gov/consumers/guides/about-fcc

Fernández, H. (2001). Internet: su problemática jurídica. Buenos Aires: Abeledo-Perrot.

Fino, D. M. (2010). Licencias Creative Commons y Web 2.o: herramientas que promueven la creación de contenidos digitales y su posición frente a los derechos de autor en Colombia. En Ramírez Cleves, G. A. (ed.). Los Blogs Jurídicos 
y la web 2.0 para la difusión y la enseñanza del Derecho (pp. 107-130). Bogotá: Universidad Externado de Colombia.

Galindo, F. (2012). Democracia, internet y gobernanza: una concreción. Revista Sequência, (65), 33-56.

Garrote, I. (2003). El derecho de autor en Internet. Los tratados de la OMPI de 1996 y la incorporación al Derecho Español de la Directiva 2001/29/CE. (2 ed). Granada: Comares.

Gobernación de Antioquia (s.f). Plan de Desarrollo 2016-2019 “Antioquia Piensa en Grande”. Recuperado de http://antioquia.gov.co/images/pdf/ ORDENANZA\%20PLAN\%20DE\%20DESARROLLO\%20DE\%20ANTIOQUIA\%20 2016-2019_FirmaEscaneada.pdf

Gobernación del Chocó (s.f). El Plan de Desarrollo Departamental del Departamento del Chocó 20162019 "Oportunidades para todas la Subregiones". Recuperado de http://choco.gov.co/apc-aafiles/396363666634383533663646466323738/ plan-de-desarrollo-departamental-del-choco-2016-2019.pdf

Gobernanza de Internet en Colombia (2014). Foro de Gobernanza de Internet. Recuperado de http:// colnodo.apc.org/destacamos.shtml?apc=1-xx$1-\& x=5682$

Hassan, A. (2005). Internet Governance: Strengths and weaknesses from business perspective. En Drake, W.J. (ed). Reforming Internet Governance: Perspectives from the working group on Internet Governance (WGIG) (pp. 117-128). New York: United Nations.

ICANN (s.f). ¿Qué hace ICANN? Recuperado de https:// www.icann.org/resources/pages/what-201202-25-es

Internet Society (2017). Reuniones de IETF. Recuperado de https://www.internetsociety.org/es/ reuniones-de-ietf
Instituto de Investigación y Debate sobre Gobernanza (2016). Un espacio intercultural, interdisciplinario y multi-actores para la reflexión sobre la gobernanza. Recuperado de: http://www.institutgouvernance.org/spip.php?article149 olang $=$ fr

Jiménez, J. (2012). Modelos de implementación de las políticas públicas en Colombia y su impacto en el bienestar social. Analecta Politica, 2 (3), 141 164. Recuperado de: https://revistas.upb.edu.co/ index.php/analecta/article/viewFile/1392/1361

Knight, J. (2007). Educación superior transfronteriza: Problemas e implicaciones referentes a la garantía de la calidad y a la acreditación. La Educación Superior en el Mundo, 1 (8), 134-145.

Kalaš et al. (2012). ICT in Primary Education. Analytical survey. En Exploring the origins, settings and initiatives (vol. 1). Moscú: UNESCO. Recuperado de http://iite. unesco.org/pics/public ations/en/ files/3214707.pdf

Kozma, R. B. y Wagner, D. A. (2005). Core indicators for monitoring and evaluation studies for ICT for education. En Trucano, M. (ed.). Monitoring and Evaluation of ICT in Education Projects, A Handbook for Developing Countries (pp. 21-31). Washington: Banco Internacional de Reconstrucción y Desarrollo. Recuperado de http://www.infodev.org/infodev -files/resource/ InfodevDocuments_9.pdf

Kurbalija, J. y Gelbstein, J. (2005). Gobernanza en Internet. Asuntos, autores y brechas. Genova: Diplofoundation.

Light, D. y Polin, D. (2010). Integrating Web 2.0 tools into the classroom: Changing the culture of learning. New York: EDC Center for Children and Technology. Recuperado de http://cct.edc. org/sites/cct.edc.org/files/publications/Integrating\%20 Web2.0.PDF 
Lopera, J., Ramírez, C., Zuluaga, M. y Ortiz, J. (2010). El método analítico como método natural. Revista Nómadas (25), 1-27.

Lucci, N. (2014). Internet Content Governance and Human Rights. Vanderbilt Journal of Entertainment \& Technology Law, 16, 809-856.

Marsden, C. (2012). Neutralidad de la red: historia, regulación y futuro IDP. Revista de Internet, Derecho y Politica, (13), 24-43.

Mayntz, R. (2001). El Estado y la sociedad civil en la gobernanza moderna. Revista Reforma y democracia, (21), 7-22.

Meneses, G. (s.f.). Colombia en los Foros Globales de Gobernanza de Internet. Recuperado de http:// www.gobernanzadeinternet.co/es/documentos/ documentos-gobernanza-de-internet

Mesa de Gobernanza de Internet (s.f.). Foro Colombiano de Gobernanza de Internet. Recuperado de http:// www.gobernanzadeinternet.co/

Mesa de Gobernanza de Internet (2016). Relatorías del 3er. Foro Colombiano de Gobernanza de Internet. Recuperado de: http://www.gobernanzadeinternet.co/es/actas

Ministerio de Educación (s.f.). Escuelas del Futuro. Recuperado de http://www.mineduc.gob.gt/ portal/contenido/menu_lateral/programas/escuelas_del_futuro/

Ministerio de Educación Nacional (s.f.a). Plan Sectorial, Documento 9. Recuperado de http://www. mineducacion.gov.co/1621/articles-293647_archivo_pdf_plansectorial.pdf

Ministerio de Educación (s.f.b). Participación ciudadana. Recuperado de http://www.mineducacion.gov. co/1759/articles-177283_recurso_1.pdf

Ministerio de las Comunicaciones (2008). Plan Nacional de Tecnologías de la Información y las Comunicaciones. Recuperado de http://www.eduteka. org/pdfdir/Colombi aPlanNacionalTIC.pdfi
Ministerio de Tecnologías de la Información y las Comunicaciones (MinTic) (2014). Informe Rendición de Cuentas 2014. Recuperado de http://www. mintic.gov.co/portal/604/articles-4323_recurso_1.pdf

Naciones Unidas (2016). Consejo de Derechos Humanos $20^{\circ}$ periodo de sesiones. Tema 3 de la agenda Promoción y protección. Recuperado de http:// ap.ohchr.org/documents/S/HRC/d_res_dec/A_ HRC_20_L13.pdf

Naciones Unidas (2016). Temas mundiales. Recuperado de http://www.un.org/es/globalissues/ governancel

Organismo especializado de las Naciones Unidas para las TIC (2006). Agenda de Túnez para la sociedad de la información. Recuperado de http://www. itu.int/net/wsis/docs2/tunis/off/6rev1-es.html

Padilla, M. (2012). El kit de la lucha en Internet. Madrid: Traficantes de sueños.

Pérez, J. y Olmos, A. (2009). Introducción. Gobernanza de Internet. Revista Telos, (80), 1-5.

Polanco, C. (2011). Políticas públicas y tic en la educación. Revista Iberoamericana de Ciencia, Tecnología y Sociedad, 6 (8), 3-18.

Programa Nacional de Nuevas Tecnologías (s.f.). Colombia aprende: la red del conocimiento. Recuperado de http://www.colombiaaprende.edu.co/html/ home/1592/article-102549.html

Rendón, H. (2012). Oficina de Innovación Educativa con Uso de las Nuevas Tecnologías, Ministerio de Educación Nacional. Recuperado de http://www.mineducacion.gov.co/cvn/1665/ articles-311722_archivo9_pdf.pdf

Rengifo, E. (2008). Un nuevo reto del Derecho en la edad de la información. Revista la Propiedad Inmaterial, (12), 1-20.

OPERA, $N^{\circ} 21 \cdot J$ ulio-Diciembre $2017 \cdot$ pp. 5-23 
Rossini, C. (2010). Green-Paper: The state and challenges of OER in Brazil: From readers to writers? Berkman Research Publication (01), 1-74.

Schmitz, C. (2005). La propiedad intelectual a la luz de los tratados de libre comercio. Santiago de Chile: Lexis Nevis.

unesCo (2002a). Technologies for Education. Potentials, Parameters, and Prospects. París UnESCO y AED. Recuperado de http://unesdoc.unesco.org/images/0011/001191/11912 9e.pdf

unesco (2002b). Open and Distance Learning: Trends, Policy and Strategy Considerations. Paris: UNESCO. Recuperado de http://unesdoc.unesco.org/ images/0012/001284/128463e.pdf

unesco (2005). Towards knowledge societies. Paris: UNESCO. Recuperado de http:// unesdoc.une sco. org/images/0014/001418/141843e.pdf
UNESCo (2014). Central Asia Symposium on ICT in Education 2014: Embracing technologies, Empowering teachers. Bangkok. Recuperado de http:// www.unescobkk.org/fileadmin/user_upload/ ict/Workshops/casie2014/CASIE_2014_Outcome_Document_English_version_.pdf

Velásquez, F. y Gómez, E. (2003). ¿Qué ha pasado con la participación ciudadana en Colombia? Bogotá: Fundación Corona. Recuperado de http://www. dhl.hegoa.ehu.es/ficheros/0000/0120/participacion_ciudadana_en_colombia.pdf

World Bank (s.f.). World Links for Development Programme (WorLD). Recuperado de http://documents.worldbank.org/curated/ en/356311468150888217/World-Links-forDevelopment-Program-WorLD 